\title{
Efferocytosis of Apoptotic Neutrophils Enhances Control of Mycobacterium tuberculosis in HIV-Coinfected Macrophages in a Myeloperoxidase-Dependent Manner
}

\author{
Anna-Maria Andersson ${ }^{a}$ Marie Larsson ${ }^{b}$ Olle Stendahl ${ }^{a}$ Robert Blomgran ${ }^{a}$ \\ ${ }^{a}$ Division of Medical Microbiology, Department of Clinical and Experimental Medicine, Faculty of Medicine and \\ Health Sciences, Linköping University, Linköping, Sweden; ${ }^{b}$ Division of Molecular Virology, Department of Clinical \\ and Experimental Medicine, Faculty of Medicine and Health Sciences, Linköping University, Linköping, Sweden
}

\author{
Keywords \\ Mycobacterium tuberculosis · HIV · Reactive oxygen species · \\ Myeloperoxidase $\cdot$ Macrophages
}

\begin{abstract}
Tuberculosis remains a big threat, with 1.6 million deaths in 2017, including 0.3 million deaths among patients with HIV. The risk of developing active disease increases considerably during an HIV coinfection. Alveolar macrophages are the first immune cells to encounter the causative agent Mycobacterium tuberculosis, but during the granuloma formation other cells are recruited in order to combat the bacteria. Here, we have investigated the effect of efferocytosis of apoptotic neutrophils by $M$. tuberculosis and HIV-coinfected macrophages in a human in vitro system. We found that the apoptotic neutrophils enhanced the control of $M$. tuberculosis in single and HIV-coinfected macrophages, and that this was dependent on myeloperoxidase (MPO) and reactive oxygen species in an autophagy-independent manner. We show that MPO remains active in the apoptotic neutrophils and can be harnessed by infected macrophages. In addition, MPO inhibition removed the suppression in M. tuberculosis growth caused by the apoptotic neutrophils. Antimycobacterial components from apoptotic neutrophils could thus in-
\end{abstract}

crease the microbicidal activity of macrophages during an $M$. tuberculosis/HIV coinfection. This cooperation between innate immune cells could thereby be a way to compensate for the impaired adaptive immunity against $M$. tuberculosis seen during a concurrent HIV infection.

(C) 2019 The Author(s)

Published by S. Karger AG, Basel

\section{Introduction}

Tuberculosis is the leading cause of death from a single infectious agent. One of the major risk factors for developing an active disease is coinfection with HIV, which affects the protective adaptive immune response. Another problem is the rise in drug-resistant tuberculosis, making the treatment more difficult and increasing mortality [1]. The causative agent Mycobacterium tuberculosis enters the lungs and predominantly infects alveolar macrophages [2]. In most instances, the microbes cause a latent infection but if the host fails to control the pathogen, an active tuberculosis infection is established. Following infection, different immune cells are recruited to the lung and a granuloma is formed to control the infection $[3,4]$. The normally well-structured tuberculosis granulomas in patients are disrupted in HIV-coinfected individuals,

\begin{tabular}{|c|c|}
\hline KARGER & $\begin{array}{l}\text { (c) } 2019 \text { The Author(s) } \\
\text { Published by S. Karger AG, Basel }\end{array}$ \\
\hline $\begin{array}{l}\text { E-Mail karger@karger.com } \\
\text { www.karger.com/jin }\end{array}$ & $\begin{array}{l}\text { This article is licensed under the Creative Commons Attribution- } \\
\text { NonCommercial-NoDerivatives } 4.0 \text { International License (CC BY- } \\
\text { NC-ND) (http://www.karger.com/Services/OpenAccessLicense). } \\
\text { Usage and distribution for commercial purposes as well as any dis- } \\
\text { tribution of modified material requires written permission. }\end{array}$ \\
\hline
\end{tabular}

Prof. Robert Blomgran

Division of Medical Microbiology, Department of Clinical and Experimental Medicine Faculty of Medicine and Health Sciences, Linköping University

SE-581 85 Linköping (Sweden)

E-Mail robert.blomgran@liu.se 
with more necrosis and a heterogeneous cellular population dominated by neutrophils and eosinophils [5].

The role of neutrophils in the defense against mycobacterium is controversial and has been implicated as both beneficial, especially during early infection, and detrimental at later stages [6-9]. However, compared to viable and apoptotic neutrophils, the necrotic phenotype leads to more severe disease progression and mycobacterial growth, especially in HIV patients [5, 10-12]. Apoptotic cells are eliminated mainly by macrophages, in a process called efferocytosis, and this can induce macrophage activation, cytokine release, and a decrease in $M$. tuberculosis growth. Depending on the study design, the mechanisms behind the increased control of M. tuberculosis in macrophages may vary, from increased phagosome maturation, to harnessing the antimicrobial content of granules from neutrophils [13-16].

Upon phagocytosis, a strong respiratory burst is triggered in neutrophils, generating reactive oxygen species (ROS) through the NADPH oxidase inside the phagosome in order to combat the ingested bacteria. Azurophilic granules containing myeloperoxidase (MPO) fuse with the phagosome and contribute to the bactericidal environment by producing hypochlorous acid ( $\mathrm{HOCl})$ from hydrogen peroxide $\left(\mathrm{H}_{2} \mathrm{O}_{2}\right)$ [17]. Although macrophages are able to produce reactive oxygen intermediates, their main route of antimicrobial action is through autophagy and lysosomal-endosomal fusion with the phagosome [18]. These innate defense mechanisms exerted by neutrophils and macrophages are inhibited by $M$. tuberculosis as a means to persist inside host cells [19-24].

It is well established that HIV coinfection impairs the activation of macrophages through the adaptive immune system by decreased antigen presentation and impaired stimulation of $M$. tuberculosis Ag-specific CD4 T cells [25-27]. Therefore, an alternative way to activate coinfected macrophages is needed. Previously, we have shown that apoptotic neutrophils are able to activate $M$. tuberculosis-infected macrophages, leading to increased proinflammatory signals and $M$. tuberculosis growth inhibition [13]. From these and other studies showing a role for the cooperation between apoptotic and viable cells in the defense against $M$. tuberculosis [14-16], we hypothesized that efferocytosis can enhance immune protection also in HIV/M. tuberculosis-coinfected cells. In our in vitro study using human cells, we show that apoptotic neutrophils enhance the control of intracellular $M$. tuberculosis both in M. tuberculosis-single and HIV-coinfected macrophages, and that this process was dependent on MPO and ROS in an autophagy-independent way.

\section{Materials and Methods}

\section{Generation of Macrophages from Monocytes}

Monocytes were isolated from buffy coats and the PBMCs were isolated through gradient centrifugation before adhesion to tissue culture flasks. Non-adherent cells were removed by extensive washing and the monocytes were cultured in DMEM supplemented with $10 \%$ pooled normal human serum for 7 days to differentiate into macrophages.

\section{Isolation of Neutrophils and Induction of Apoptosis}

Polymorphonuclear cells were separated through gradient centrifugation from peripheral blood. The red blood cells were lysed by hypotonic shock followed by KRG washing of the neutrophils. The neutrophils were then resuspended in RPMI containing $2 \mathrm{mM}$ of L-glutamine and 10\% heat-inactivated FBS, and left for around $20 \mathrm{~h}$ at $37^{\circ} \mathrm{C}$ for spontaneous apoptosis. Staining with Annexin V $(\mathrm{AV})$ and propidium iodide (PI) showed that around $70 \%$ were apoptotic after this incubation; $64.8 \%( \pm 3.2)$ were apoptotic $(\mathrm{AV}+)$ and $4.8 \%( \pm 0.5)$ were late apoptotic $(\mathrm{AV}+\mathrm{PI}+)$. Prior to experiment, the apoptotic neutrophils were washed once before being resuspended in suitable medium for coculture with macrophages. For some experiments the neutrophils were stained with PKH26 red fluorescent membrane labeling kit (MINI26 Sigma) prior to apoptosis, according to the manufacturer's instructions.

\section{Preparation of M. tuberculosis}

M. tuberculosis $\mathrm{H} 37 \mathrm{Rv}$ was cultured for 2 weeks in $M$. tuberculosis medium (Middlebrook $7 \mathrm{H} 9$ with $0.05 \%$ Tween- $80,0.5 \%$ glycerol, and 10\% ADC enrichment) and passaged 1 week before use. For GFP-expressing $M$. tuberculosis the medium was supplemented with $20 \mu \mathrm{g} / \mathrm{mL}$ of kanamycin, and for luciferase-expressing $M$. tuberculosis $100 \mu \mathrm{g} / \mathrm{mL}$ of hygromycin was used. For infection, the bacteria were prepared as previously described [28].

HIV and M. tuberculosis Coinfection

Macrophages were infected with $0.06 \mathrm{ng} / \mathrm{mL}$ HIV-1BaL (Lot p4238), produced as previously described [28], for 1 week prior to M. tuberculosis infection at MOI =1-5. After M. tuberculosis infection the macrophages were incubated with apoptotic neutrophils (1:2) for different time points, depending on the experiment.

\section{Flow Cytometry and Confocal Microscopy}

GFP-expressing M. tuberculosis-infected macrophages, seeded in 96-well plates or on cover slips, and treated with labelled apoptotic neutrophils, were stained with $75 \mathrm{~nm}$ of LysoTracker Deep Red (cat. No. L12492; Life Technologies) to visualize acidic organelles for $2 \mathrm{~h}$ prior to fixation with 4\% PFA. These cells were then analyzed either by flow cytometry or confocal microscopy. Some cells were treated with $100 \mathrm{nM}$ of bafilomycin A1 (from Streptomyces griseus, Sigma Aldrich) $1 \mathrm{~h}$ before and during the infection as a negative control for acidification.

MPO staining was performed after fixation of macrophages that had been infected and exposed to apoptotic neutrophils for 30 $\min$ to $24 \mathrm{~h}$. The cells were permeabilized with $0.1 \%$ saponin for 30 min followed by wash and staining with the primary antibody polyclonal rabbit anti-human MPO (A0398, DAKO) diluted 1:400 for $1 \mathrm{~h}$ at room temperature. After washing with PBS, the secondary antibody goat anti-rabbit AF647 (A21244, Life Technologies) diluted 1:400 was added for $30 \mathrm{~min}$ at $37^{\circ} \mathrm{C}$. Following washing, 
DAPI diluted 1:100 was added for $15 \mathrm{~min}$ at room temperature before the cover slips were washed and mounted.

All cover slips were analyzed in a LSM 700 Zeiss upright confocal microscope with a plan apochromat $63 x$, NA 1.40 objective. Images were acquired with Zen software and all samples were observed in a blinded fashion where 50-300 phagosomes/sample were examined. Image brightness and contrast were adjusted equally with Photoshop for representative micrographs only, after the completion of the blinded analysis. Pseudo colors were chosen for optimal display of the results.

\section{Cytokine Measurement}

Supernatants from infected macrophages treated with apoptotic neutrophils were analyzed for cytokines through cytometric bead array analysis, performed according to the manufacturer's instructions (BD Biosciences). Data were analyzed using Kaluza software (Beckman Coulter, Fullerton, CA, USA).

\section{M. tuberculosis Growth Assay}

For the M. tuberculosis growth assay, macrophages were infected with luciferase-expressing M. tuberculosis for $1.5 \mathrm{~h}$, extracellular bacteria washed off with media, followed by addition of apoptotic neutrophils that had been pretreated with $500 \mu \mathrm{M}$ of the MPO inhibitor 4-aminobenzoic hydrazide (ABAH; A41909, Sigma) for $1 \mathrm{~h}$. The growth of $M$. tuberculosis was measured as described previously [29] after 5 days and compared to the day 0 values (phagocytosis).

\section{Western Blot}

Macrophages were infected for $1.5 \mathrm{~h}$ prior to washing and the addition of apoptotic neutrophils for a total of $24 \mathrm{~h}$ when the cells were collected and Western blot was performed as previously described [28]. The antibodies were: rabbit monoclonal anti-LC3B (D11; cat. No. 3868, Cell Signaling), mouse monoclonal antiSQSTM1 D-3 (cat. No. sc-28359, Santa Cruz Biotechnology), and mouse monoclonal anti- $\beta$-actin (clone AC-74; cat. No. A2228, Sigma-Aldrich). The dilutions of the antibodies were 1:5,000 for LC3, $1: 2,000$ for SQSTM1, and 1:10,000 for $\beta$-actin. The secondary antibodies polyclonal goat anti-rabbit or anti-mouse immunoglobulins/HRP (Dako Cytomation) were diluted 1:2,000 for LC3 and SQSTM1, and 1:10,000 for $\beta$-actin. Band intensities were quantified using ImageJ.

\section{ROS Measurement}

The probe CM-H2DCFDA (C6827, Invitrogen) was used for general oxidative stress detection in macrophages. $5 \mu \mathrm{M}$ was added together with $M$. tuberculosis for $1 \mathrm{~h}$ at $37^{\circ} \mathrm{C}$ with a wash before and after, prior to the addition of apoptotic neutrophils. Measurements were performed $1 \mathrm{~h}$ after uptake of apoptotic neutrophils that had been pretreated with $500 \mu \mathrm{M} \mathrm{ABAH}$ for $1 \mathrm{~h}$ and washed prior to addition to the macrophages. The ROS from macrophages or apoptotic or viable neutrophils were measured as chemiluminescence using the substrate luminol $(20 \mu \mathrm{g} / \mathrm{mL}$; Sigma-Aldrich) upon ABAH inhibition $(0-1,000 \mu \mathrm{M})$ and $0.1 \mu \mathrm{M}$ PMA (SigmaAldrich) or $0.01 \mu \mathrm{M}$ fMLP (Sigma-Aldrich) stimulation.

\section{Statistical Analysis}

All statistical analyses were performed with GraphPad prism software. The data were analyzed using repeated-measures ANO$\mathrm{VA}$, with the post hoc Bonferroni's multiple comparison test (unless otherwise indicated). $p$ values $<0.05$ were considered significant.

\section{Results}

\section{Apoptotic Neutrophils Inhibit Growth of}

M. tuberculosis in Macrophages

It is not known if efferocytosis of apoptotic neutrophils can enhance the capacity of HIV-coinfected macrophages to control $M$. tuberculosis. Therefore, apoptotic neutrophils were added to $M$. tuberculosis-single and $\mathrm{HIV} / M$. tuberculosis-coinfected macrophages, and the bacterial load was assessed 5 days after infection. When investigating the growth of $M$. tuberculosis, we washed away the non-phagocytosed bacteria after infection before adding apoptotic neutrophils in order to start the experiment with the same bacterial load in the different cells. Consistent with our previous results [13], M. tuberculosis growth was decreased by apoptotic neutrophils after 5 days (Fig. 1a, b). This growth inhibition effect was significant both when analyzing the intracellular $M$. tuberculosis (macrophage lysate) and the total bacteria (combined intra- plus extracellular contribution; Fig. 1a, b). Furthermore, there was no difference between single and coinfected macrophages, indicating that apoptotic neutrophils have the capacity to inhibit $M$. tuberculosis growth also in HIV-infected macrophages.

To confirm that apoptotic neutrophils were taken up by the infected/coinfected macrophages to the same extent, the uptake of apoptotic neutrophils was tracked. Already after $1 \mathrm{~h}, 50 \%$ of the macrophages had taken up apoptotic neutrophils (ApoN+), which further increased, reaching around $80 \%$ at $24 \mathrm{~h}$ (Fig. $2 \mathrm{a}$ ). Infected and coinfected macrophages both ingested apoptotic neutrophils at a similar rate. Furthermore, the colocalization between apoptotic neutrophils and $M$. tuberculosis also increased with time (Fig. 2b). We also assessed M. tuberculosis uptake in order to evaluate if apoptotic neutrophils affected the phagocytosis of $M$. tuberculosis. Apoptotic neutrophils were added to infected macrophages without removing non-phagocytosed bacteria, thereby allowing further phagocytosis of $M$. tuberculosis. After $4 \mathrm{~h}$ of stimulation with apoptotic neutrophils (i.e., when $>60 \%$ of the macrophages contained apoptotic neutrophils), we observed a $20 \%$ increase in $M$. tuberculosis-GFP+ macrophages that also contained apoptotic neutrophils, compared to those that had been stimulated with but did not contain apoptotic neutrophils (Fig. 2c). This was further confirmed by an increase in the total $M$. tuberculosis-GFP signal (MFI) in macrophages with apoptotic neutrophils from 2.2 to 3.8 in single infected and 2.0 to 3.6 in HIVcoinfected macrophages (Fig. 2d). 


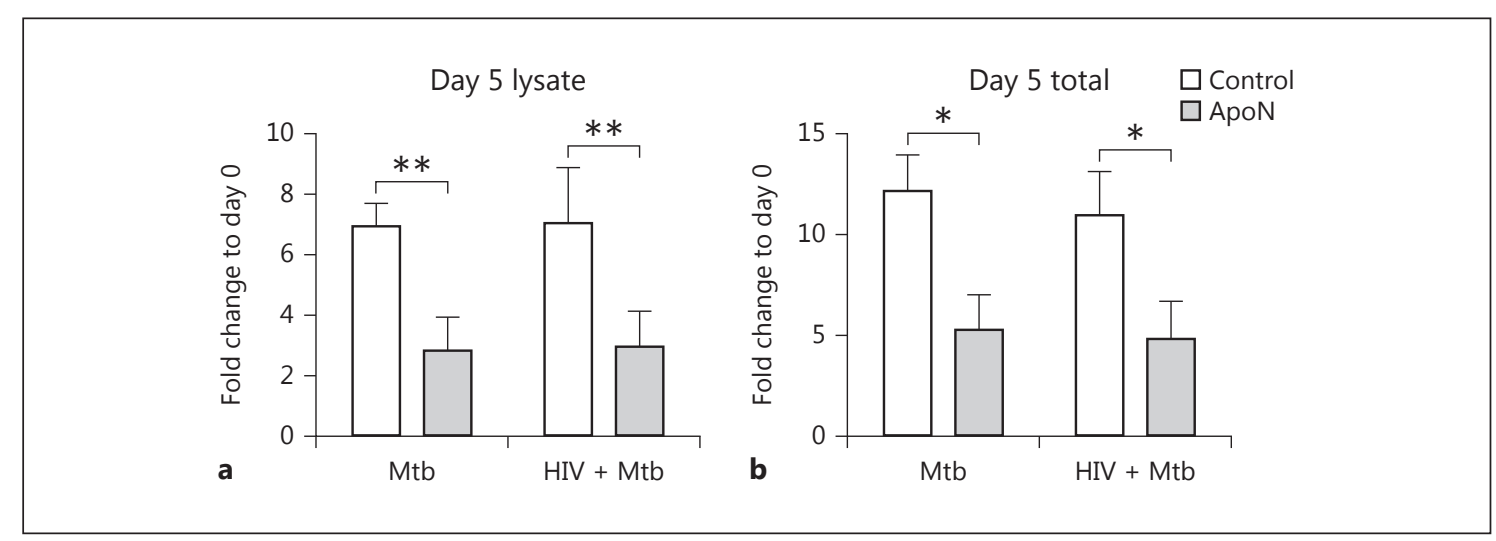

Fig. 1. Apoptotic neutrophils decreased M. tuberculosis growth both in single and HIV-coinfected macrophages. Human macrophages were preinfected with/without HIV for 7 days before infection with $M$. tuberculosis $(\mathrm{Mtb}, \mathrm{MOI}=1)$ for $1.5 \mathrm{~h}$. After a wash, apoptotic neutrophils (ApoN) were added for 5 days and the sig- nals from luciferase expressing $M$. tuberculosis in cell lysates (a) and total bacteria (supernatant + lysate; b) were measured. Data are the mean \pm SEM from 4 independent experiments. ${ }^{*} p<0.05$, ** $p<0.01$, using repeated-measures ANOVA.

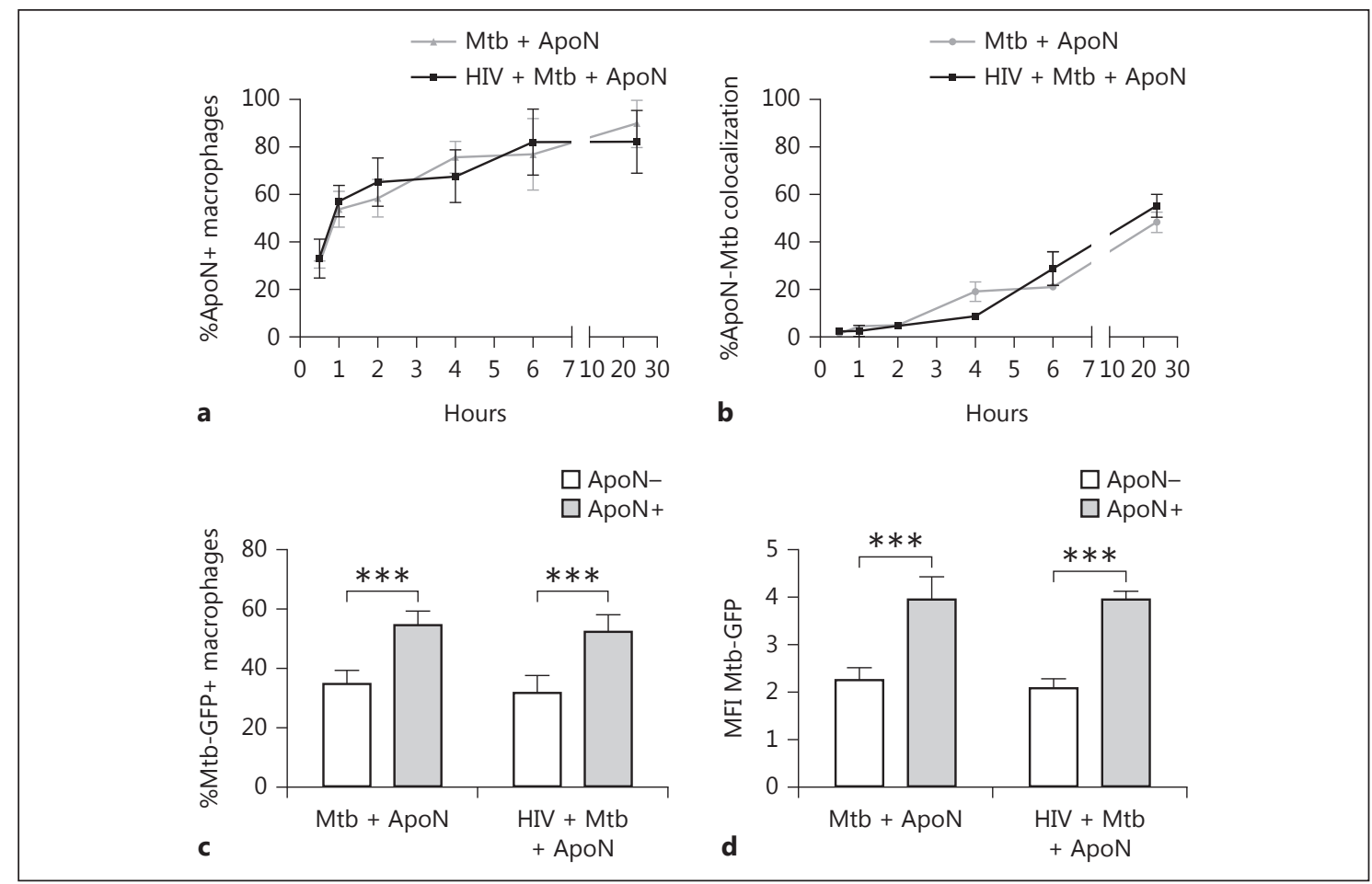

Fig. 2. Uptake of apoptotic neutrophils by macrophages is time dependent and causes an increase in bacterial phagocytosis. The percentage of macrophages containing apoptotic neutrophils (ApoN+; a) and percentage of colocalization of apoptotic neutrophils to $M$. tuberculosis phagosomes (b), at the indicated time points, as quantified by microscopy. The macrophages were first infected with HIV followed by M. tuberculosis (Mtb, MOI = 2) infection for $1.5 \mathrm{~h}$, and extracellular bacteria were washed away before stimulation with apoptotic neutrophils for up to $24 \mathrm{~h}$. The graph shows the mean \pm SEM from 2 independent experiments. c, d Flow cytometry analysis revealed that phagocytosis of $M$. tuberculosis (\% Mtb-GFP+ macrophages and MFI Mtb-GFP) was increased in infected macrophages containing apoptotic neutrophils $(\mathrm{ApoN}+)$ compared to those that were exposed but did not ingest apoptotic neutrophils (ApoN-). Data are the mean \pm SEM from 6 independent experiments, where the macrophages were $M$. tuberculosis infected $(\mathrm{MOI}=2)$ for $2 \mathrm{~h}$ followed by apoptotic neutrophil stimulation for $4 \mathrm{~h}$ without washing away the bacteria. *** $p<0.001$, using repeated-measures ANOVA. 


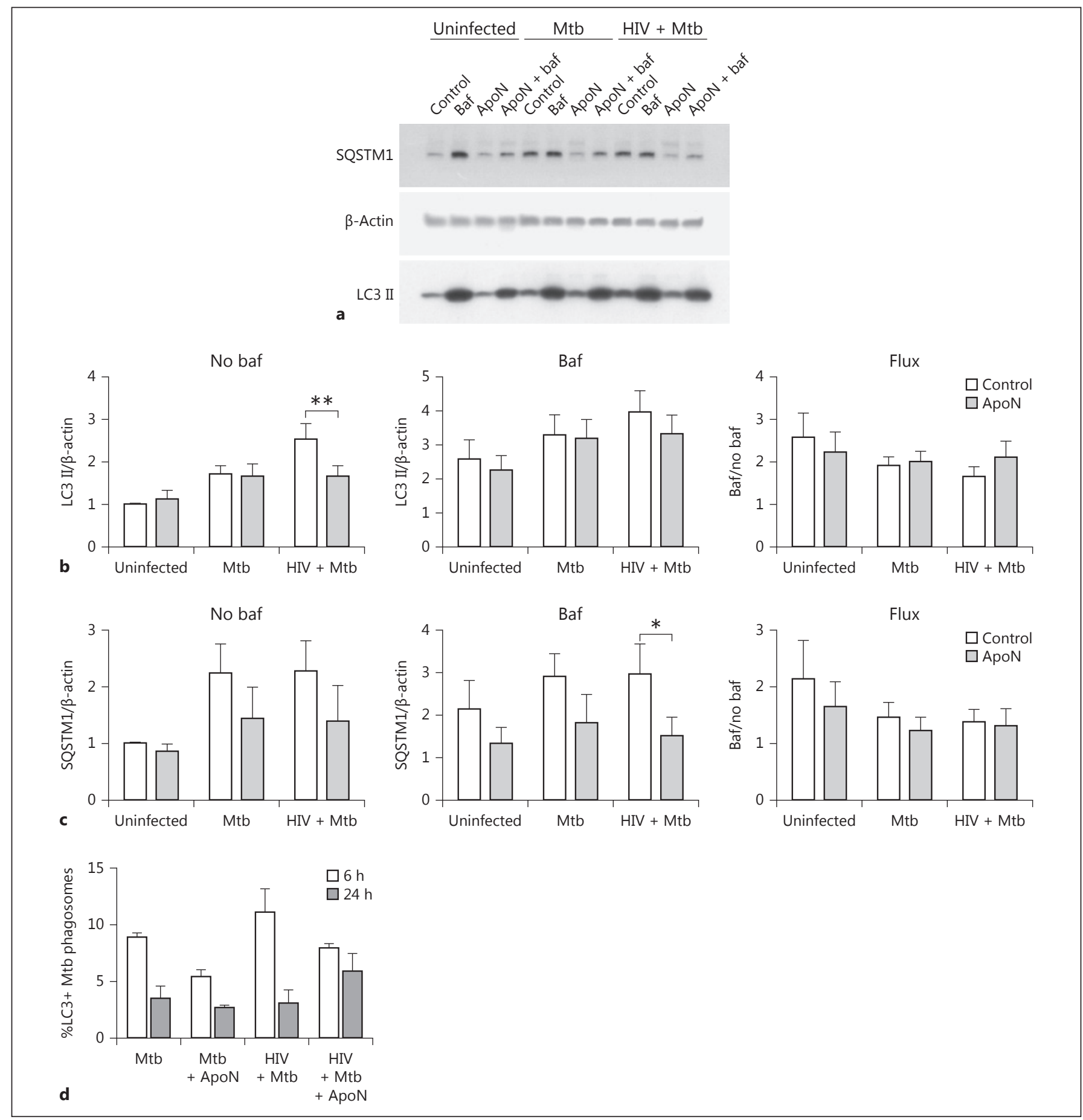

Fig. 3. Apoptotic neutrophils do not cause any changes in autophagic flux. a Representative immunoblots showing the autophagy markers LC3B and SQSTM1 (p62), with their $\beta$-actin loading controls. The autophagy markers LC3 II (b) and SQSTM1 (c) were quantified from Western blots and normalized to their respective $\beta$-actin control and presented as the ratio over uninfected macrophages without apoptotic neutrophils (ApoN). The macrophages were infected with $M$. tuberculosis (Mtb, MOI = 5) for $1.5 \mathrm{~h}$, washed, and stimulated with apoptotic neutrophils for $22.5 \mathrm{~h}$. "Baf" indicates that the macrophages were pretreated with bafilomycin for $1 \mathrm{~h}$ prior to infection, while the graphs named "Flux" show the autophagic flux (i.e., samples with baf/samples without baf). Data are shown as the mean \pm SEM with * $p<0.05$ and ${ }^{* *} p<0.01$ using repeated-measures $\operatorname{ANOVA}(n=6)$. $\mathbf{d}$ The percentage of LC3 colocalization to $M$. tuberculosis was quantified by microscopy after prior HIV infection and $1.5 \mathrm{~h}$ of $M$. tuberculosis infection $(\mathrm{MOI}=2)$ followed by washing and stimulation with apoptotic neutrophils for $4.5 \mathrm{~h}$ ( $6 \mathrm{~h}$ in total) or $22.5 \mathrm{~h}(24 \mathrm{~h}$ in total). Data are the mean \pm SEM from 4 independent experiments. 


\section{Apoptotic Neutrophils Do Not Cause Changes in Autophagic Flux}

Since autophagy has been suggested to have a protective role during M. tuberculosis infection [30-32], we evaluated if efferocytosis of apoptotic neutrophils can stimulate autophagy or drive the autophagic flux. We studied the autophagy proteins LC3 II (Fig. 3a, b) and SQSTM1 (Fig. 3a, c) in whole cell lysates, and bafilomycin was used to inhibit the last steps of autophagy in order to evaluate the total accumulation of autophagosomes. The autophagic flux can be determined by the ratio of total autophagosomes formed (with bafilomycin) and those formed and being degraded (without bafilomycin). Values $\leq 1$ indicated no flux, and values $>1$ indicated autophagic flux. Apoptotic neutrophils did not affect the autophagic flux (Fig. 3a-c), rather they decreased formation of autophagosomes, most noticeably for SQSTM1 with bafilomycin and with the same tendency without bafilomycin. Colocalization of LC3 with M. tuberculosis at 6 and $24 \mathrm{~h}$ postinfection further confirmed this. There was a tendency of decreased autophagosome formation at $6 \mathrm{~h}$ in single and coinfected macrophages when stimulated with apoptotic neutrophils (Fig. 3d). The LC3-M. tuberculosis colocalization levels decreased after $24 \mathrm{~h}$, but to a lesser extent in the infected mac-
Fig. 4. Apoptotic neutrophils decrease acidification of $M$. tuberculosis phagosomes. a Representative micrographs of LysoTracker (LT) colocalization to apoptotic neutrophils (ApoN) or M. tuberculosis (Mtb) phagosomes in infected macrophages. Green, Mtb; red, LT; blue, ApoN. Macrophages were infected with $M$. tuberculosis for $2 \mathrm{~h}(\mathrm{MOI}=1)$, stimulated with apoptotic neutrophils for $4 \mathrm{~h}$, with the addition of the probe LysoTracker (LT) for the last $2 \mathrm{~h}$ before fixation and colocalization studies by confocal microscopy. The percentage of colocalization of $M$. tuberculosis to LT (b) and apoptotic neutrophils to LT (c) for 6 donors, showing the mean \pm SEM. ${ }^{*} p<0.05$ using repeated-measures ANOVA.

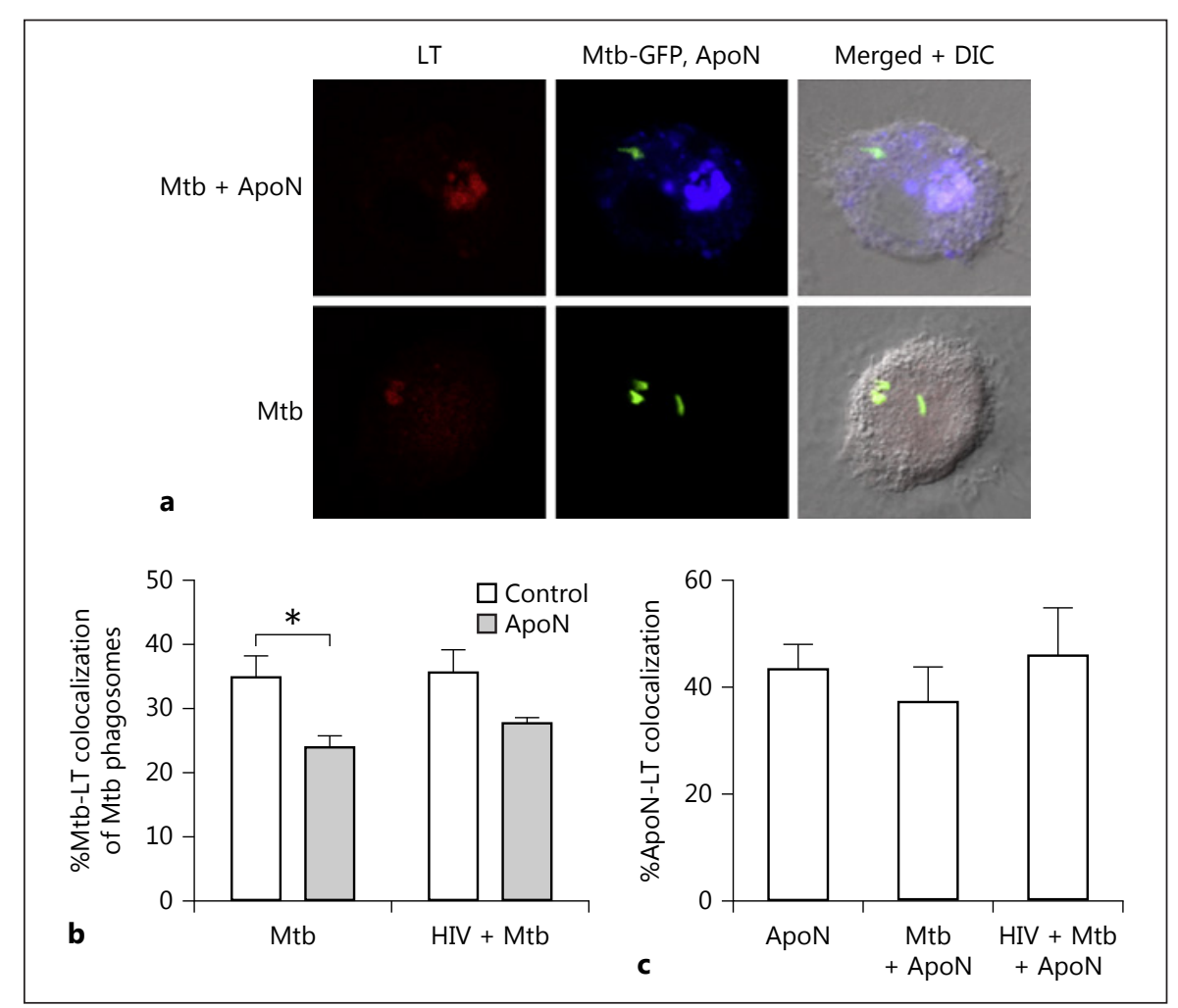

Fig. 5. MPO, which is active even after apoptosis, is present in macrophages which have ingested apoptotic neutrophils (ApoN). ROS production by apoptotic neutrophils was measured through chemiluminescence upon stimulation with PMA (a) or fMLP (b), showing the mean from 3 independent experiments. a MPO was inhibited by $30 \mathrm{~min}$ of preincubation with increasing concentrations of ABAH (as indicated) before luminol and PMA was added to the apoptotic neutrophils. b Freshly isolated neutrophils (PMN) and apoptotic neutrophils were stimulated with fMLP and their ROS production measured in the presence of luminol. c Percentage of MPO+ macrophages at the indicated time points, as quantified by microscopy (d). e Percentage of MPO colocalization with
M. tuberculosis (Mtb) phagosomes at the indicated time points. The macrophages were first infected with HIV followed by $M$. $t u$ berculosis $(\mathrm{MOI}=2)$ infection for $1.5 \mathrm{~h}$, washed, and stimulated with apoptotic neutrophils for up to $24 \mathrm{~h}$. The graph shows the mean \pm SEM from 2 independent experiments. $d$ The microscopy images visualize the macrophages by DIC, while apoptotic neutrophils are shown in yellow, $M$. tuberculosis-GFP in green, MPO in red, and DAPI in blue. The first row shows the cells 30 min after the addition of apoptotic neutrophils, the second after $4 \mathrm{~h}$, and the last one after $24 \mathrm{~h}$. The last column shows infected macrophages that have not been stimulated with ApoN but have been stained for MPO.

(For figure see next page.) 


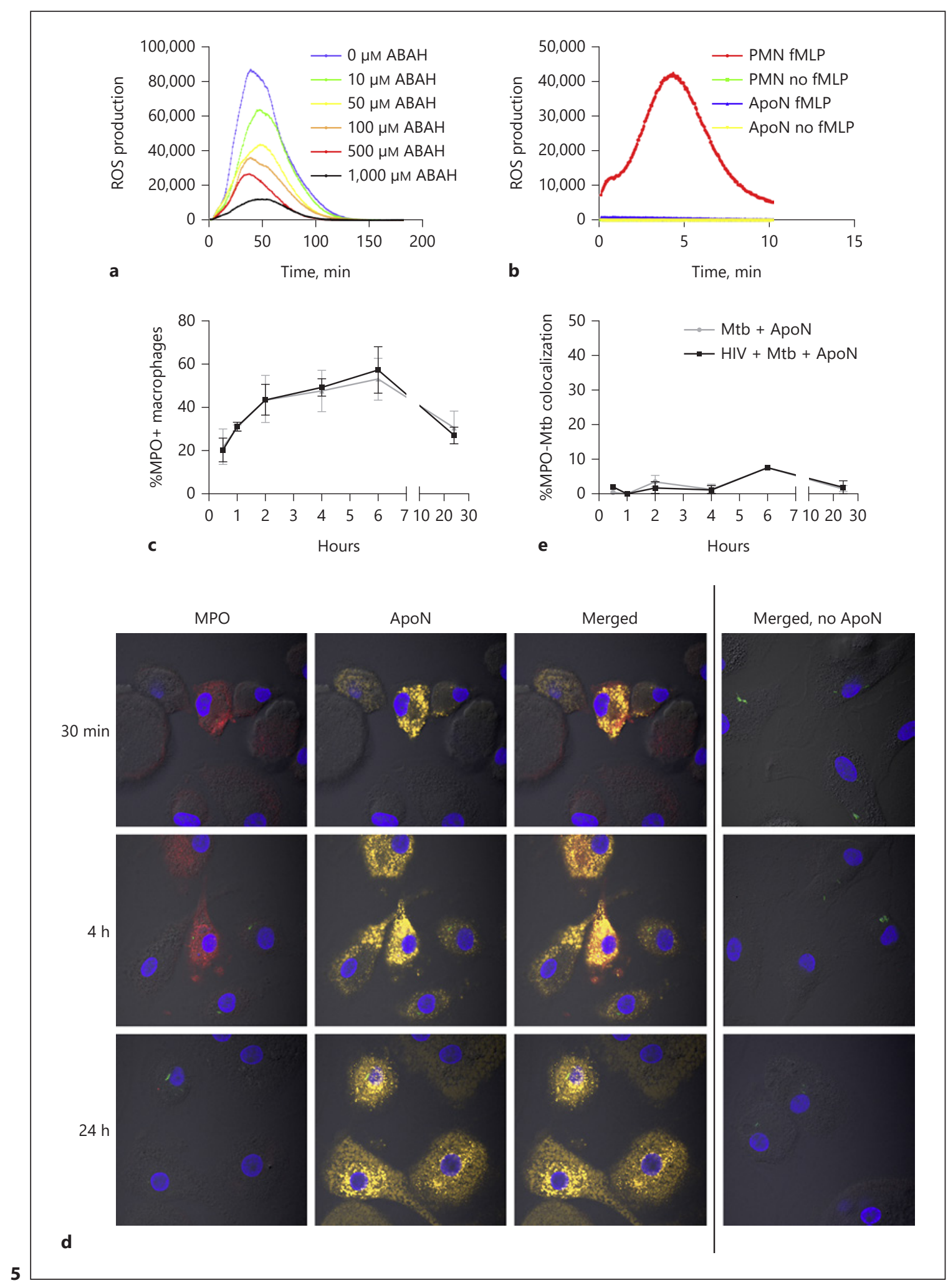




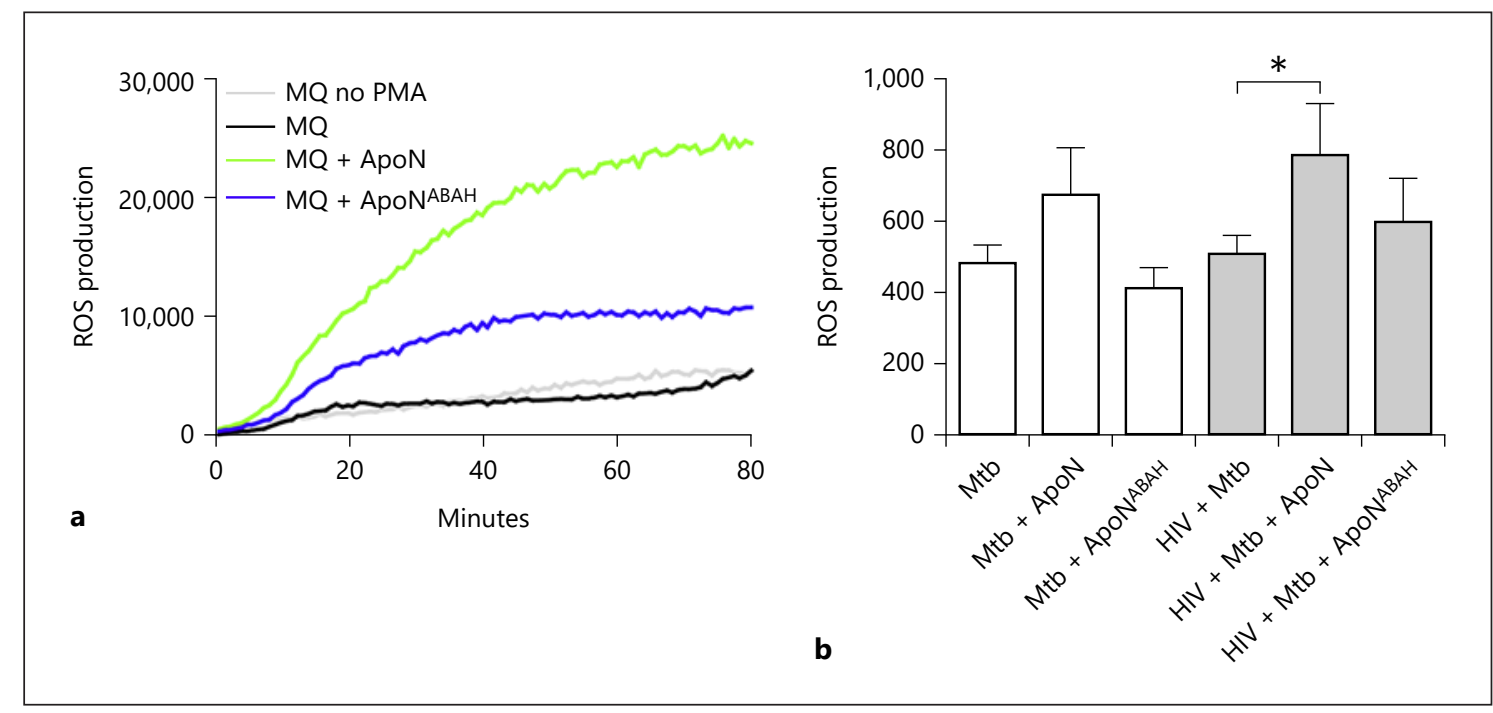

Fig. 6. Apoptotic neutrophils increase ROS production in macrophages in an MPO-dependent manner. a ROS production in macrophages was measured through chemiluminescence upon stimulation with PMA. Untreated apoptotic neutrophils (ApoN) and those treated with $\mathrm{ABAH}\left(\mathrm{ApoN} \mathrm{N}^{\mathrm{ABH}}\right)$ were added to macrophages for $24 \mathrm{~h}$, followed by washing and PMA stimulation in the presence of HRP. The graph shows the mean from 3 independent experiments. b ROS production measured with the probe CMH2DCFDA in infected macrophages stimulated with apoptotic neutrophils for $1 \mathrm{~h}$. Apoptotic neutrophils were untreated or treated with the MPO inhibitor ABAH (ApoN $\left.{ }^{\mathrm{ABAH}}\right)$ and washed, before being added to the macrophages. The graph shows the mean \pm SEM from 5 independent experiments. ${ }^{*} p<0.05$ using repeatedmeasures ANOVA. rophages stimulated with apoptotic neutrophils, indicating less degradation of LC3 in those macrophages. Together, these results suggest that autophagosome formation is not directly involved when apoptotic neutrophils decrease the growth of M. tuberculosis in macrophages.

Lysosome Fusion with M. tuberculosis Phagosomes Is Further Inhibited by Apoptotic Neutrophils

To further analyze phagolysosome fusion during efferocytosis, we assessed the acidification of the $M$. tuberculosis phagosomes using the probe LysoTracker (Fig. 4a). There was a decreased colocalization between $M$. tuberculosis and LysoTracker in macrophages with apoptotic neutrophils (Fig. 4b). This indicated that acidification and maturation of the $M$. tuberculosis phagosomes were not involved in the decreased M. tuberculosis viability caused by the apoptotic neutrophils. Moreover, we observed a colocalization of around $40 \%$ between apoptotic neutrophils and LysoTracker in uninfected and infected macrophages (Fig. 4c). This was a higher colocalization than that seen between M. tuberculosis and LysoTracker, both without (approx. 35\%) and with apoptotic neutrophils (approx. $25 \%$; Fig. 4b). Flow cytometry data showed a significant $(p<0.01)$ overall increase in LysoTracker signal (MFI) in macrophages containing apoptotic neutrophils (30.1 \pm 4.1 in single and $31.1 \pm 5.5$ in coinfected macrophages, respectively) compared to those that did not $(17.1 \pm 1.5$ in single and $18.1 \pm 3.0$ in coinfected macrophages, respectively). However, from the microscopy data it is evident that this acidification was clearly concentrated to apoptotic neutrophils and did not localize to M. tuberculosis.

MPO, Which Is Active after Apoptosis, Is Present in

Macrophages That Have Phagocytosed Apoptotic

Neutrophils

Neutrophils can generate an oxidative burst owing to its high expression of the NADPH oxidase, generating superoxide anions $\left(\mathrm{O}_{2}^{-}\right)$, which dismutates to hydrogen peroxide $\left(\mathrm{H}_{2} \mathrm{O}_{2}\right)$, which is further catalyzed into the more bactericidal $\mathrm{HOCl}$ by the azurophilic granular protein MPO [33, 34]. To investigate if this system is still intact in apoptotic neutrophils, we measured the ROS production using a luminol-enhanced chemiluminescence assay. Activation of apoptotic neutrophils with the phorbol ester PMA triggered a strong ROS response (Fig. 5a), whereas a receptor-dependent stimulus fMLP only induced an ROS response in live neutrophils, but not in apoptotic neutrophils (Fig. 5b). The PMA-induced ROS response by apoptotic neutrophils was additionally confirmed to be MPO dependent using the irreversible 
Fig. 7. The suppressed M. tuberculosis growth caused by apoptotic neutrophils is rescued through MPO inhibition. Apoptotic neutrophils (ApoN) were treated with $500 \mu \mathrm{M}$ of $\mathrm{ABAH}\left(\mathrm{ApoN}^{\mathrm{ABAH}}\right)$ for $1 \mathrm{~h}$ prior to washing and added to $M$. tuberculosis (Mtb, MOI = 1) infected (a) and HIVcoinfected (b) macrophages for 5 days. The intracellular growth is shown as the mean \pm SEM from 5 independent experiments. ${ }^{*} p<0.05,{ }^{* *} p<0.01$, using repeated-measures ANOVA.

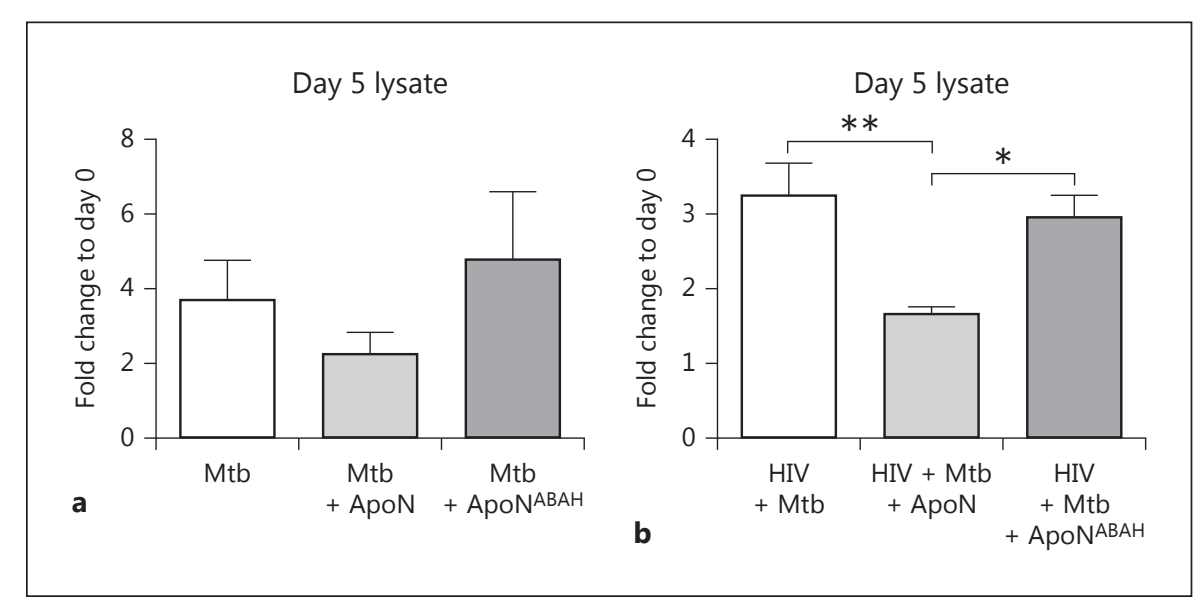

MPO-inhibitor ABAH (Fig. 5a). There was a time-dependent increase of MPO inside the macrophages that had ingested apoptotic neutrophils, which was not seen in the absence of apoptotic neutrophils, confirming that the neutrophils were the source of MPO (Fig. 5c, d). The increase in MPO was correlated to the early kinetics observed for uptake of apoptotic neutrophils (Fig. 2a). However, in contrast to the uptake of apoptotic neutrophils, there was a decreased MPO inside the macrophages after $24 \mathrm{~h}$ (Fig. 5c, d). This indicated that the effect of MPO in efferocytosing macrophages could be transient. MPO seemed to be spread in the cytoplasm of macrophages upon uptake of apoptotic neutrophils. The colocalization of MPO to M. tuberculosis phagosomes remained below $10 \%$ after $24 \mathrm{~h}$ (Fig. 5e).

\section{ROS Production in Macrophages Is Increased upon}

Phagocytosis of Apoptotic Neutrophils

We further observed an increased ROS production in uninfected (Fig. 6a) as well as HIV-coinfected macrophages after uptake of apoptotic neutrophils (Fig. 6b). Evidence for the involvement of MPO for the increased ROS production in macrophages was further supported by $\mathrm{ABAH}$ pretreatment of the apoptotic neutrophils, which decreased ROS production (Fig. 6a, b). Of interest is that the ROS experiments with uninfected macrophages (Fig. 6a) was performed $24 \mathrm{~h}$ after uptake of apoptotic neutrophils (at which time point there was less discernable MPO staining in macrophages), indicating that MPO could mediate a long-lasting functional response. These results showed that the complex for ROS generation in apoptotic neutrophils was sequestered by macrophages through efferocytosis and could enhance the antimicrobial capacity of infected macrophages.

Myeloperoxidase Inhibits M. tuberculosis Growth during HIV Coinfection
M. tuberculosis Growth Inhibition Caused by the Apoptotic Neutrophils Is MPO Dependent

Since autophagy or phagosome maturation were not affected by uptake of apoptotic neutrophils, we next explored whether ROS and MPO from apoptotic neutrophils support M. tuberculosis killing. ABAH was therefore used to inhibit the MPO activity of the apoptotic neutrophils before they were added to the infected macrophages. When irreversibly inhibiting MPO with $\mathrm{ABAH}$, the $M$. tuberculosis growth control caused by apoptotic neutrophils was reversed, although only significant in the HIVcoinfected macrophages (Fig. 7a, b). These results indicate that MPO and ROS are involved in the enhanced control of M. tuberculosis in macrophages after uptake of apoptotic neutrophils.

\section{Apoptotic Neutrophils Do Not Increase the}

Proinflammatory Response in M. tuberculosis-

Infected Macrophages

In order to determine whether the decrease in $M$. $t u$ berculosis growth caused by the apoptotic neutrophils was due to an induced proinflammatory response by the macrophages, we measured the production of the proinflammatory cytokines IL- $1 \beta$, TNF- $\alpha$, and IL-6 (Fig. 8a-c). We found that the apoptotic neutrophils caused a decrease in IL- $1 \beta$ production in both $M$. tuberculosis-single and HIV-coinfected macrophages. This was partly restored by the inhibition of MPO with ABAH. Furthermore, TNF- $\alpha$ was reduced in $M$. tuberculosis-infected macrophages upon stimulation with apoptotic neutrophils, with the same tendency for IL-6. These results suggest that the apoptotic neutrophils do not stimulate a proinflammatory cytokine response in macrophages infected with live virulent $M$. tuberculosis. 


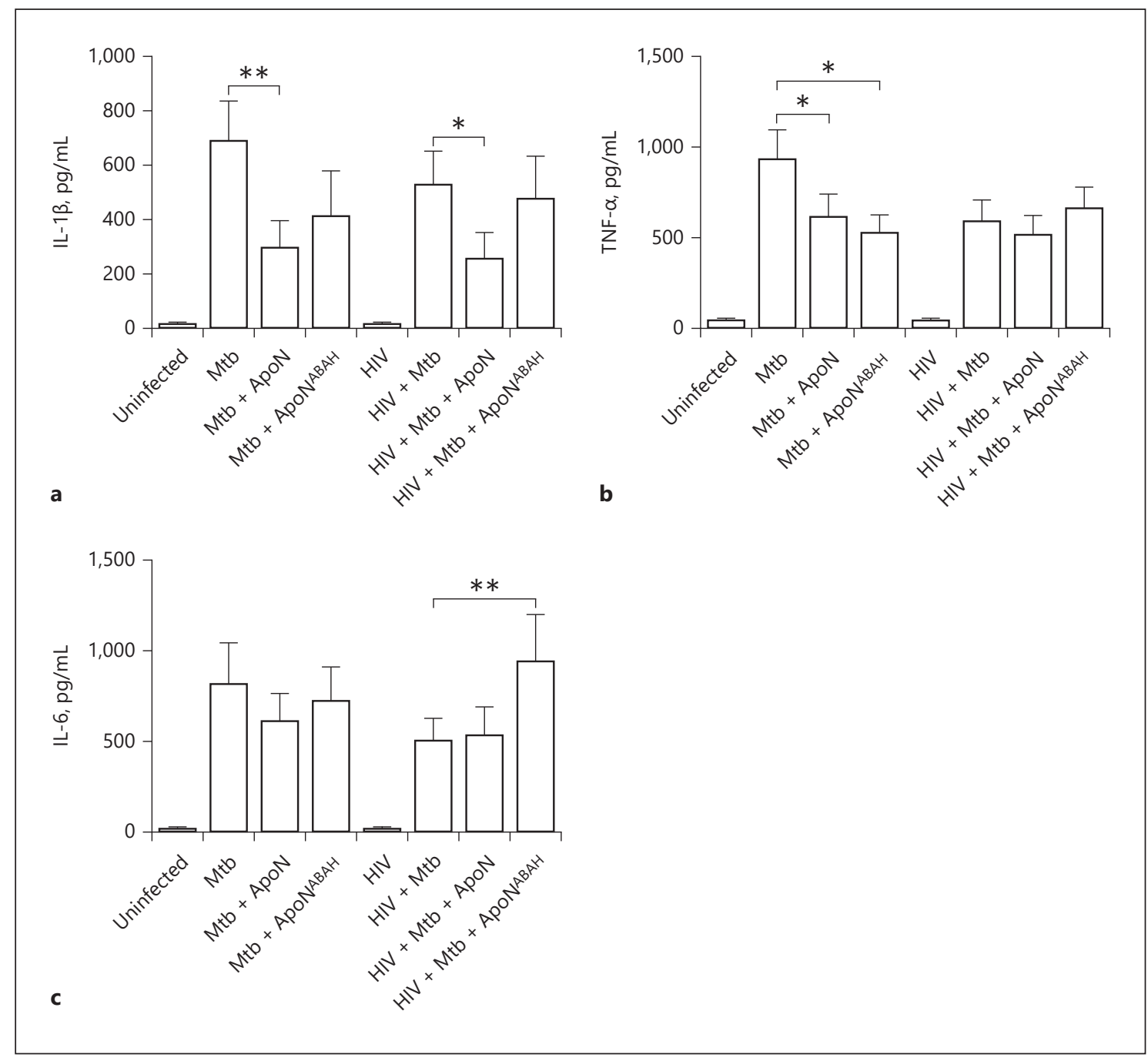

Fig. 8. Apoptotic neutrophils decrease the proinflammatory response in infected macrophages. IL-1 $\beta$ (a), TNF- $\alpha(\mathbf{b})$, and IL-6 (c) were measured in the supernatants of $M$. tuberculosis and HIVcoinfected macrophages. Apoptotic neutrophils (ApoN) were treated with $500 \mu \mathrm{M}$ of $\mathrm{ABAH}\left(\mathrm{ApoN}^{\mathrm{ABAH}}\right)$ for $1 \mathrm{~h}$ prior to washing and added to the infected macrophages. The macrophages were

\section{Discussion}

M. tuberculosis Ag-specific CD4 T cells are depleted or are impaired in HIV/M. tuberculosis-coinfected individuals $[35,36]$, and we recently showed that HIV/M. tuberculosis coinfection skews these effector cells into a suppressive phenotype that fails to control M. tuberculosis in macrophages $[25,37]$. As the adaptive immune response cannot support control of M. tuberculosis during HIV/M. tuberculosis coinfection, we here investigated if the function of coinfected macrophages can be boosted in other infected with $M$. tuberculosis $(\mathrm{Mtb}, \mathrm{MOI}=2)$ for $1.5 \mathrm{~h}$ prior to stimulation with apoptotic neutrophils for $22.5 \mathrm{~h}$. Data are the mean \pm SEM from 11 independent experiments. ${ }^{*} p<0.05,{ }^{* *} p<$ 0.01 , using repeated-measures ANOVA with Dunnett's multiple comparison test.

ways. Using an in vitro model, we showed a pronounced time-dependent uptake of the apoptotic neutrophils by coinfected macrophages. This uptake was associated with a reduction in $M$. tuberculosis growth, as only observed by others during $M$. tuberculosis-single infection [13-16]. We further established that the decreased M. tuberculosis growth was MPO dependent and that production of ROS was enhanced in efferocytosing macrophages, suggesting a role for ROS and MPO in the defense against $M$. tuberculosis. This cooperation between neutrophils and macrophages could thus be a way to compensate for the im- 
pairment of the adaptive immunity against $M$. tuberculosis seen during HIV coinfection.

In contrast to other reports $[14,16]$, we did not observe any changes in autophagic flux or increased phagosomal maturation caused by the apoptotic cells. In contrast, we detected a higher percentage of LysoTracker colocalization to apoptotic neutrophils in macrophages than to the bacterial phagosomes. This is in concordance with other observations showing that apoptotic cells are swiftly cleared by macrophages in order to avoid secondary necrosis [38-41], whereas $M$. tuberculosis is able to inhibit phagosomal maturation $[42,43]$. The additional decrease in maturation of $M$. tuberculosis-containing phagosomes in macrophages containing apoptotic neutrophils could be due to the increase in $\mathrm{pH}$ upon ROS production. A study supporting this theory showed that M1 macrophages exhibit higher phagosomal $\mathrm{pH}$ along with delayed phagosome-lysosome fusion compared to M2 macrophages, in part due to the increased proton consumption during intraphagosomal ROS production in M1 macrophages [44]. Neutrophil phagosomes normally have a higher $\mathrm{pH}$ than macrophage phagosomes, since neutrophils generate a strong oxidative burst, while the macrophages act mainly through lysosome fusion with the phagosomes [45-48]. However, since we observed very little colocalization between $M$. tuberculosis and apoptotic neutrophils or MPO, their effect was most likely not limited to the phagosome but could occur in the cytosol. There are scenarios when $M$. tuberculosis does not necessarily need to be in the cytosol for them to be affected by reactions occurring in the cytosol or reactions taking place in compartments with close proximity to the M. tuberculosis-containing phagosome. For instance, as part of their phagosomal escape strategy virulent $M$. tuberculosis express the ESX-1 type VII secretion system leading to pore formation and damage to the phagosomal membrane [49]. This inadvertently puts the bacterium also in contact with host defense mechanisms or components of the cytosol, as in this case the accumulation of MPO in macrophages that have ingested apoptotic neutrophils, leading to enhanced control of $M$. tuberculosis.

The antimicrobial activity of ROS and MPO against mycobacteria has been studied extensively, with earlier studies suggesting a role in bacterial killing [50-53], while more recent studies suggest that $M$. tuberculosis is only sensitive to endogenous ROS, generated within the bacteria $[54,55]$. M. tuberculosis has several defense mechanisms against ROS, one being katG catalase-peroxidase which transforms $\mathrm{H}_{2} \mathrm{O}_{2}$ to oxygen and water.
Isoniazid-resistant $M$. tuberculosis, lacking katG, is more susceptible to $\mathrm{H}_{2} \mathrm{O}_{2}[21,22]$. As our experiments were performed with virulent $M$. tuberculosis $\mathrm{H} 37 \mathrm{Rv}$ with wild-type kat $G$, this indicates that the efferocytosismediated ROS production is at a level beyond the threshold that M. tuberculosis can neutralize, and they therefore succumb to killing. Alternatively, the overall ROS production inside the macrophage triggers signaling cascades involved in killing of intracellular M. tuberculosis. Indeed, ROS have been implicated as signaling mediators, able to modulate phagocytosis, gene expression, and promoting a proinflammatory response [56, 57]. Although other studies have shown a role for MPO in stimulating TNF secretion by macrophages $[58,59]$, in this study using live virulent $M$. tuberculosis we did not find an induction of a proinflammatory response. In contrast to our earlier findings with $\gamma$-irradiated inactive M. tuberculosis H37Rv [13], we show that efferocytosis of apoptotic neutrophils by macrophages infected with live virulent $M$. tuberculosis $\mathrm{H} 37 \mathrm{Rv}$ decrease the proinflammatory response. The reason for this could be that growth restriction of $M$. tuberculosis during efferocytosis reduce the stimulatory effect of M. tuberculosis. This would suggest that neutrophil granules are able to boost the immune response of macrophages coinfected with HIV and M. tuberculosis, without causing additional proinflammatory activation. In this study we have used HIV-1 BaL, which is a CCR5 tropic virus. HIV-1 BaL has the ability to infect $\mathrm{T}$ cells and dendritic cells in mucosa in a manner similar to the HIV isolates found to establish HIV infection in primary HIV-infected individuals [60], so the effects we found should be of relevance in vivo.

In conclusion we have found that efferocytosis of apoptotic neutrophils can inhibit $M$. tuberculosis growth in M. tuberculosis-single and HIV-coinfected macrophages. The decrease in M. tuberculosis growth in coinfected macrophages was MPO dependent, and we suggest that its effect is mediated by ROS and MPO rather than autophagy and lysosome fusion. Our study clearly shows the importance of cooperation between cells of the innate immune system and that apoptotic neutrophils can contribute to an enhanced killing of $M$. tuberculosis inside macrophages during HIV coinfection. Stimulating efferocytosis of apoptotic neutrophils or the uptake of MPO via neutrophil extracellular traps could therefore be a strategy to compensate for the impaired adaptive immune response during HIV infection. 


\section{Acknowledgements}

We thank Julian Bess and the Biological Products Core of the AIDS and Cancer Virus Program (Leidos Biomedical Research Inc., Frederick National Laboratory, Frederick, MD, USA) for providing HIV-1 virus preparations.

\section{Statement of Ethics}

The authors have no ethical conflicts to disclose. Blood, buffy coats, and normal human serum from heathy donors were obtained from the blood bank at Linköping University Hospital, who had given written consent for research use of the donated blood in accordance with the Declaration of Helsinki. Thus, this study did not require a specific ethical approval according to paragraph 4 of the Swedish law (2003:460) on Ethical Conduct in Human Research.

\section{Disclosure Statement}

The authors have no conflicts of interest to declare.

\section{Funding Sources}

This work was supported by Swedish Research Council Grant 2017-05617 (to R.B.) and Grant 348-2013-6588 (to O.S.), Swedish Heart-Lung Foundation Grant 2014-0578 (to O.S.), and Grants 2016-0431, 2016-0719, and 2018-0615 (to R.B.), the Stiftelsen Clas Groschinskys Minnesfond (to R.B.), and Swedish Society of Medicine Grant SLS-499971 (to R.B.).

\section{Author Contributions}

A.-M.A., R.B., and O.S. conceived and designed the experiments; M.L. provided HIV; A.-M.A. performed the experiments and analyzed the data; A.-M.A. and R.B. wrote the paper, with inputs from all authors.

\section{References}

1 World Health Organization. Global tuberculosis report 2018. WHO; 2018. Available from: http://www.who.int/tb/publications/ global_report/en/

2 Cohen SB, Gern BH, Delahaye JL, Adams KN, Plumlee CR, Winkler JK, et al. Alveolar Macrophages Provide an Early Mycobacterium tuberculosis Niche and Initiate Dissemination. Cell Host Microbe. 2018 Sep;24(3):439446.e4.

3 Ndlovu H, Marakalala MJ. Granulomas and inflammation: host-directed therapies for tuberculosis. Front Immunol. 2016 Oct; 7:434.

4 Kiran D, Podell BK, Chambers M, Basaraba RJ. Host-directed therapy targeting the Mycobacterium tuberculosis granuloma: a review. Semin Immunopathol. 2016 Mar;38(2): $167-83$.

5 de Noronha AL, Báfica A, Nogueira L, Barral A, Barral-Netto M. Lung granulomas from Mycobacterium tuberculosis/HIV-1 co-infected patients display decreased in situ TNF production. Pathol Res Pract. 2008;204(3): $155-61$.

6 Yang CT, Cambier CJ, Davis JM, Hall CJ, Crosier PS, Ramakrishnan L. Neutrophils exert protection in the early tuberculous granuloma by oxidative killing of mycobacteria phagocytosed from infected macrophages. Cell Host Microbe. 2012 Sep;12(3):301-12.

7 Lowe DM, Redford PS, Wilkinson RJ, O’Garra A, Martineau AR. Neutrophils in tuberculosis: friend or foe? Trends Immunol. 2012 Jan; $33(1): 14-25$.
8 Eruslanov EB, Lyadova IV, Kondratieva TK, Majorov KB, Scheglov IV, Orlova MO, et al. Neutrophil responses to Mycobacterium tuberculosis infection in genetically susceptible and resistant mice. Infect Immun. 2005 Mar; 73(3):1744-53.

9 Blomgran R, Ernst JD. Lung neutrophils facilitate activation of naive antigen-specific CD4+ T cells during Mycobacterium tuberculosis infection. J Immunol. 2011 Jun; 186(12):7110-9.

10 Lowe DM, Bangani N, Goliath R, Kampmann B, Wilkinson KA, Wilkinson RJ, et al. Effect of antiretroviral therapy on HIV-mediated impairment of the neutrophil antimycobacterial response. Ann Am Thorac Soc. 2015 Nov; 12(11):1627-37.

11 Dallenga T, Repnik U, Corleis B, Eich J, Reimer R, Griffiths GW, et al. M. tuberculosisinduced necrosis of infected neutrophils promotes bacterial growth following phagocytosis by macrophages. Cell Host Microbe. 2017 Oct;22(4):519-530.e3.

12 Lowe DM, Demaret J, Bangani N, Nakiwala JK, Goliath R, Wilkinson KA, et al. Differential effect of viable versus necrotic neutrophils on Mycobacterium tuberculosis growth and cytokine induction in whole blood. Front Immunol. 2018 Apr;9:903.

13 Andersson H, Andersson B, Eklund D, Ngoh E, Persson A, Svensson K, et al. Apoptotic neutrophils augment the inflammatory response to Mycobacterium tuberculosis infection in human macrophages. PLoS One. 2014 Jul;9(7):e101514.
14 Martin CJ, Booty MG, Rosebrock TR, NunesAlves C, Desjardins DM, Keren I, et al. Efferocytosis is an innate antibacterial mechanism. Cell Host Microbe. 2012 Sep;12(3): 289-300.

15 Tan BH, Meinken C, Bastian M, Bruns H, Legaspi A, Ochoa MT, et al. Macrophages acquire neutrophil granules for antimicrobial activity against intracellular pathogens. J Immunol. 2006 Aug;177(3):1864-71.

16 Hartman ML, Kornfeld H. Interactions between naïve and infected macrophages reduce Mycobacterium tuberculosis viability. PLoS One. 2011;6(11):e27972.

17 Dupré-Crochet S, Erard M, Nüße O. ROS production in phagocytes: why, when, and where? J Leukoc Biol. 2013 Oct;94(4):657-70.

18 Weiss G, Schaible UE. Macrophage defense mechanisms against intracellular bacteria. Immunol Rev. 2015 Mar;264(1):182-203.

19 May ME, Spagnuolo PJ. Evidence for activation of a respiratory burst in the interaction of human neutrophils with Mycobacterium tuberculosis. Infect Immun. 1987 Sep;55(9): 2304-7.

20 Deretic V, Singh S, Master S, Harris J, Roberts E, Kyei G, et al. Mycobacterium tuberculosis inhibition of phagolysosome biogenesis and autophagy as a host defence mechanism. Cell Microbiol. 2006 May;8(5):719-27.

$21 \mathrm{Ng} \mathrm{VH}$, Cox JS, Sousa AO, MacMicking JD, McKinney JD. Role of KatG catalase-peroxidase in mycobacterial pathogenesis: countering the phagocyte oxidative burst. Mol Microbiol. 2004 Jun;52(5):1291-302. 
22 Ehrt S, Schnappinger D. Mycobacterial survival strategies in the phagosome: defence against host stresses. Cell Microbiol. 2009 Aug;11(8):1170-8.

23 Liu CH, Liu H, Ge B. Innate immunity in tuberculosis: host defense vs pathogen evasion. Cell Mol Immunol. 2017 Dec;14(12):963-75.

24 Hussain Bhat K, Mukhopadhyay S. Macrophage takeover and the host-bacilli interplay during tuberculosis. Future Microbiol. 2015; 10(5):853-72.

25 Singh SK, Andersson AM, Ellegård R, Lindestam Arlehamn CS, Sette A, Larsson M, et al. HIV interferes with Mycobacterium tuberculosis antigen presentation in human dendritic cells. Am J Pathol. 2016 Dec;186(12):308393.

26 Patel NR, Zhu J, Tachado SD, Zhang J, Wan Z, Saukkonen J, et al. HIV impairs TNF-a mediated macrophage apoptotic response to Mycobacterium tuberculosis. J Immunol. 2007 Nov;179(10):6973-80.

27 Geldmacher C, Ngwenyama N, Schuetz A, Petrovas C, Reither K, Heeregrave EJ, et al. Preferential infection and depletion of $\mathrm{Myco}$ bacterium tuberculosis-specific CD4 T cells after HIV-1 infection. J Exp Med. 2010 Dec; 207(13):2869-81.

28 Andersson AM, Andersson B, Lorell C, Raffetseder J, Larsson M, Blomgran R. Autophagy induction targeting mTORC1 enhances Mycobacterium tuberculosis replication in HIV co-infected human macrophages. Sci Rep. 2016 Jun;6(1):28171.

29 Eklund D, Welin A, Schön T, Stendahl O, Huygen K, Lerm M. Validation of a mediumthroughput method for evaluation of intracellular growth of Mycobacterium tuberculosis. Clin Vaccine Immunol. 2010 Apr;17(4):513-

30 Gutierrez MG, Master SS, Singh SB, Taylor GA, Colombo MI, Deretic V. Autophagy is a defense mechanism inhibiting BCG and Mycobacterium tuberculosis survival in infected macrophages. Cell. 2004 Dec;119(6):753-66.

31 Zhang Q, Sun J, Wang Y, He W, Wang L, Zheng Y, et al. Antimycobacterial and antiinflammatory mechanisms of baicalin via induced autophagy in macrophages infected with Mycobacterium tuberculosis. Front Microbiol. 2017 Nov;8:2142.

32 Campbell GR, Spector SA. Vitamin D inhibits human immunodeficiency virus type 1 and Mycobacterium tuberculosis infection in macrophages through the induction of autophagy. PLoS Pathog. 2012;8(5):e1002689.

33 Aratani Y. Myeloperoxidase: its role for host defense, inflammation, and neutrophil function. Arch Biochem Biophys. 2018 Feb;640: $47-52$.

34 Lincoln JA, Lefkowitz DL, Cain T, Castro A, Mills KC, Lefkowitz SS, et al. Exogenous myeloperoxidase enhances bacterial phagocytosis and intracellular killing by macrophages. Infect Immun. 1995 Aug;63(8):3042-7.
35 Devalraju KP, Neela VS, Ramaseri SS, Chaudhury A, Van A, Krovvidi SS, et al. IL-17 and IL-22 production in HIV+ individuals with latent and active tuberculosis. BMC Infect Dis. 2018 Jul;18(1):321.

36 Murray LW, Satti I, Meyerowitz J, Jones M, Willberg CB, Ussher JE, et al. Human Immunodeficiency Virus Infection Impairs Th1 and Th17 Mycobacterium tuberculosis-Specific T-Cell Responses. J Infect Dis. 2018 May; 217(11):1782-92.

37 Singh SK, Larsson M, Schön T, Stendahl O, Blomgran R. HIV Interferes with the Dendritic Cell-T Cell Axis of Macrophage Activation by Shifting Mycobacterium tuberculosis-Specific CD4 T Cells into a Dysfunctional Phenotype. J Immunol. 2019 Feb;202(3):816-26.

38 Martin CJ, Peters KN, Behar SM. Macrophages clean up: efferocytosis and microbial control. Curr Opin Microbiol. 2014 Feb;17: $17-23$.

39 Garcia-Aguilar T, Espinosa-Cueto P, Magallanes-Puebla A, Mancilla $\mathrm{R}$. The mannose receptor is involved in the phagocytosis of mycobacteria-induced apoptotic cells. J Immunol Res. 2016;2016:3845247.

40 Arandjelovic S, Ravichandran KS. Phagocytosis of apoptotic cells in homeostasis. Nat Immunol. 2015 Sep;16(9):907-17.

41 Poon IK, Lucas CD, Rossi AG, Ravichandran KS. Apoptotic cell clearance: basic biology and therapeutic potential. Nat Rev Immunol. 2014 Mar; 14(3):166-80.

42 Armstrong JA, Hart PD. Response of cultured macrophages to Mycobacterium tuberculosis, with observations on fusion of lysosomes with phagosomes. J Exp Med. 1971 Sep;134(3 Pt 1): 713-40.

43 Vergne I, Chua J, Deretic V. Tuberculosis toxin blocking phagosome maturation inhibits a novel Ca2+/calmodulin-PI3K hVPS34 cascade. J Exp Med. 2003 Aug;198(4):653-9.

44 Canton J, Khezri R, Glogauer M, Grinstein S. Contrasting phagosome $\mathrm{pH}$ regulation and maturation in human M1 and M2 macrophages. Mol Biol Cell. 2014 Nov;25(21):333041.

45 Levine AP, Duchen MR, de Villiers S, Rich PR, Segal AW. Alkalinity of neutrophil phagocytic vacuoles is modulated by HVCN1 and has consequences for myeloperoxidase activity. PLoS One. 2015 Apr;10(4):e0125906.

46 Segal AW, Geisow M, Garcia R, Harper A, Miller R. The respiratory burst of phagocytic cells is associated with a rise in vacuolar $\mathrm{pH}$. Nature. 1981 Apr;290(5805):406-9.

47 Haas A. The phagosome: compartment with a license to kill. Traffic. 2007 Apr;8(4):31130 .

Innate Immun 2020;12:235-247
48 Nüsse O. Biochemistry of the phagosome: the challenge to study a transient organelle. ScientificWorldJournal. 2011;11:2364-81.

49 Simeone R, Sayes F, Song O, Gröschel MI, Brodin P, Brosch R, et al. Cytosolic access of Mycobacterium tuberculosis: critical impact of phagosomal acidification control and demonstration of occurrence in vivo. PLoS Pathog. 2015 Feb;11(2):e1004650.

50 Jackett PS, Aber VR, Lowrie DB. Virulence of Mycobacterium tuberculosis and susceptibility to peroxidative killing systems. J Gen Microbiol. 1978 Aug;107(2):273-8.

51 Klebanoff SJ, Shepard CC. Toxic effect of the peroxidase-hydrogen peroxide-halide antimicrobial system on Mycobacterium leprae. Infect Immun. 1984 May;44(2):534-6.

52 Laochumroonvorapong P, Paul S, Manca C, Freedman VH, Kaplan G. Mycobacterial growth and sensitivity to $\mathrm{H} 2 \mathrm{O} 2$ killing in human monocytes in vitro. Infect Immun. 1997 Nov;65(11):4850-7.

53 Borelli V, Banfi E, Perrotta MG, Zabucchi G. Myeloperoxidase exerts microbicidal activity against Mycobacterium tuberculosis. Infect Immun. 1999 Aug;67(8):4149-52.

54 Tyagi P, Dharmaraja AT, Bhaskar A, Chakrapani H, Singh A. Mycobacterium tuberculosis has diminished capacity to counteract redox stress induced by elevated levels of endogenous superoxide. Free Radic Biol Med. 2015 Jul;84:344-54.

55 Libardo MD, de la Fuente-Nuñez C, Anand K, Krishnamoorthy G, Kaiser P, Pringle SC, et al. Phagosomal Copper-Promoted Oxidative Attack on Intracellular Mycobacterium tuberculosis. ACS Infect Dis. 2018 Nov;4(11): 1623-34.

56 Yang CS, Shin DM, Lee HM, Son JW, Lee SJ, Akira S, et al. ASK1-p38 MAPK-p47phox activation is essential for inflammatory responses during tuberculosis via TLR2-ROS signalling. Cell Microbiol. 2008 Mar;10(3) $741-54$.

57 Fialkow L, Wang Y, Downey GP. Reactive oxygen and nitrogen species as signaling molecules regulating neutrophil function. Free Radic Biol Med. 2007 Jan;42(2):153-64.

58 Lefkowitz DL, Mills KC, Moguilevsky N, Bollen A, Vaz A, Lefkowitz SS. Regulation of macrophage function by human recombinant myeloperoxidase. Immunol Lett. 1993 Apr; 36(1):43-9.

59 Grattendick K, Stuart R, Roberts E, Lincoln J, Lefkowitz SS, Bollen A, et al. Alveolar macrophage activation by myeloperoxidase: a model for exacerbation of lung inflammation. Am J Respir Cell Mol Biol. 2002 Jun;26(6):716-22.

60 Merbah M, Arakelyan A, Edmonds T, Ochsenbauer C, Kappes JC, Shattock RJ, et al. HIV-1 expressing the envelopes of transmitted/founder or control/reference viruses have similar infection patterns of CD4 T-cells in human cervical tissue ex vivo. PLoS One. 2012;7(12):e50839. 